\title{
Combined Factors Effect of Menstrual Cycle and Background Noise on Visual Inspection Task Performance: a Simulation-based Task
}

\author{
Titis Wijayanto ${ }^{1), 3)}$, Yutaka Tochihara ${ }^{2)}$, Andi R. Wijaya ${ }^{3)}$ and Setia Hermawati ${ }^{3)}$ \\ 1) Department of Ergonomics, Graduate School of Design, Kyushu University \\ 2) Department of Ergonomics, Faculty of Design, Kyushu University \\ 3) Department of Mechanical and Industrial Engineering, Gadjah Mada University, Indonesia
}

\begin{abstract}
It is well known that women are physiologically and psychologically influenced by the menstrual cycle. In addition, the presence of background noise may affect task performance. So far, it has proven difficult to describe how the menstrual cycle and background noise affect task performance; some researchers have found an increment of performance during menstruation or during the presence of noise, others found performance deterioration, while other still have reported no dominant effect either of the menstrual cycle in performance or of the presence of noise. However, no study to date has investigated the combinational effect between the menstrual cycle and the presence of background noise in task performance. Therefore, the purpose of this study was to examine the combined factor effect of menstrual cycle and background noise on visual inspection task performance indices by Signal Detection Theory (SDT) metrics: sensitivity index $\left(d^{\prime}\right)$ and response criteria index $(\beta)$. For this purpose, ten healthy female students $(21.5 \pm 1.08$ years $)$ with a regular menstrual cycle participated in this study. A VDT-based visual inspection task was used for the experiment in $3 \times 2$ factorial designs. Two factors, menstrual phase, pre-menstruation (PMS), menstruation (M), and post-menstruation (PM) and background noise, with $80 \mathrm{~dB}(\mathrm{~A})$ background noise and without noise, were analyzed as the main factors in this study. The results concluded that the sensitivity index $\left(d^{\prime}\right)$ of SDT was affected in all the menstrual cycle conditions $(p<0.01)$ and the presence of background noise $(p<0.05)$ but no combinational effect of menstrual cycle and background noise was found in this study. On the other hand, no significant effect was observed in the subject's tendency in visual inspection, shown by $\beta$ along the menstrual cycle and the presence of background noise. According to the response criteria for each individual subject, the presence of noise affected the tendency of some subjects in detecting the object and making decision during the visual inspection task. J Physiol Anthropol 28(6): 253-259, 2009 http://www.jstage.jst.go.jp/browse/jpa2
\end{abstract}

[DOI: 10.2114/jpa26.28.253]

Keywords: menstrual cycle, background noise, visual inspection task performance, Signal Detection Theory (SDT)

\section{Introduction}

The quality inspection task is one of the important work elements in manufacturing industry and has been recognized as a matter of primary importance for customer satisfaction. Gallwey (1998) points out that many losses would be suffered due to non-conformity a product produced as the result of poor quality inspection. In order to attain a good quality inspection system and high productivity of the manufacturing system, many attempts have been made to develop automated visual inspection as a replacement for the traditional inspection system of manual human inspection. However, in many cases the sensory capabilities of an automated manufacturing system are underdeveloped and the quality inspection process is still carried out manually and visually. This kind of quality inspection includes recognition, defect detection, and decisionmaking to be done manually and visually without any automation process. The inspection performances are then characterized by the error rate or in more detail by measuring false alarms and the rate of misses.

In Indonesia, as the number of women participating in the labor force and workplaces has increased, many manufacturing industries employ women as operators of quality inspection. To achieve a better quality inspection system that involves women as operators, visual inspection performance involving the cognitive abilities should be maintained. On the other hand, both the physical and mental stress involved in an operator job might have negative effects and there are still many problems related to female health that need to be paid attention to, including the remaining issues of menstrual cycles. 
There is the fact that physical discomfort and emotional symptoms related to the menstrual cycle are experienced by women, and it is also believed that this cycle affects their capacity to work (Kasamatsu et al., 2002). A number of research studies have been carried out to investigate the effect of the menstrual cycle on the cognitive process and have indicated different results. Several studies reported that the menstrual cycle affected visual impairment, which might be associated with the fluctuation of endogenous hormone levels and sex steroid levels (Ward et al., 1978; Philips and Sherwin, 1992). Rosenberg and Park (2001) reported that no significant difference in spatial ability was found but there was improvement of verbal working memory, which then was associated with a period of high estrogen levels. A study on visual inspection task performance during the menstrual cycle was also investigated by Nakatani et al. (1993) using Signal Detection Theory (SDT) and the results showed that the menstrual cycle did not significantly affect cognitive, physiological, and subjective performance. Thus it was considered that the performance of women during the menstrual cycle might be varied by individual experience during menstrual cycle period which might also result in different cognitive performance during visual inspection task.

In addition to the menstrual cycle effect on cognitive performance, the physical environment such as background noise, vibration, thermal stressor, and lighting might affect cognitive performance (Parsons, 2000). Gramopadhye and Wilson (1997) point out that visual inspection performance was primarily affected by the presence of background noise in the work environment. In such a manufacturing system, background noise from the machinery cannot be avoided even though the operators wear ear plugs as protection. Several studies reported that the effect of background noise indicated different results on performance. Gawron's (1982) reviews reported that knowledge of the noise effect on performance was still inconclusive, with some research reporting background noise facilitated the performance, other research reporting the opposite, while other research still there was no effect on performance. We noted that background noise on had impaired some tasks such as focused tasks (Smith, 1991), memory task (Gomes et al., 1999), and detection task (Smith, 1988). According to those results we hypothesized that the presence of background noise during visual inspection would result in performance deterioration.

Although substantial research has been performed in evaluating cognitive performance influenced by the menstrual cycle or the physical environment, it has proven difficult to conclude whether the female physical condition during the menstrual cycle or the presence of background noise in working environment affects on cognitive performance. Moreover, there were a number of studies to date in evaluating the menstrual cycle effect or background noise effect on visual inspection task performance, but most studies investigated the single effects of a single stressor alone. Little has been investigated of their combinational effect on performance and we found no study to date that has investigated the combination effect between the menstrual cycle and background noise in working environment on visual inspection performance. Warner and Heimstra (1972) suggested that background noise intensity did not act alone in affecting cognitive performance. The interactive effects of these stresses, when combined, could be quite varied.

In this study, we examined the combined factor effect of the menstrual cycle and the presence of background noise on visual inspection task performance. The visual inspection task performance was studied from a signal detection theory (SDT) standpoint, and it was hypothesized that the combinational effect of the menstrual cycle and background noise would affect the visual inspection task.

\section{Methods}

\section{Subjects}

Ten healthy female students $(21.5 \pm 1.08$ years $)$ with a regular menstrual cycle participated in this study without any incentive. Subjects were requested to confirm that they had a regular menstrual cycle length between 26 and 30 days for at least 6 months prior to participation in this study and that they had no history of taking hormonal contraceptives, were not pregnant, and were not smokers. No special screening test on hearing and visual impairment were made; however, through questionnaires given to the subjects to check subject backgrounds, none of them claimed to have physical hearing defects, and five subjects wore glasses. A within-subject design was employed to avoid any individual differences effect.

\section{Procedures}

This study was designed using a randomized $3 \times 2$ factorial design with two factors (independent variables) to be investigated. The factors were menstrual phase: pre-menstruation (PMS), menstruation (Mens), and post-menstruation (PM), and background noise, with $80 \mathrm{~dB}(\mathrm{~A})$ background noise and without noise condition (the background noise in the environment was maintained below $55 \mathrm{~dB}(\mathrm{~A})$ during the experiment). Visual inspection tasks under two noise conditions were conducted in a day for each menstrual phase; the order of the presence of noise was randomized for each subject. The usage of this factorial design enabled observation of main effect and interaction among factors to be investigated. Background noise produced from a saw machine sound was presented to subjects using a speaker. The noise was played through a CD player and the level was set at $80 \mathrm{~dB}(\mathrm{~A})$. The design of the experiment is shown in Table 1.

All subjects were asked to inspect 300 pictures of objects (composed of $10 \%$ pictures of defective objects and $90 \%$ pictures of non-defective objects or a signal-to-noise ratio of $1: 9$ ) displayed randomly on a visual display terminal (VDT) as shown in Fig. 1. Each picture was randomly displayed between intervals of 2-4 seconds after subjects made decisions whether the picture was a defective object by pressing a 
Table 1 Experimental design

\begin{tabular}{cll}
\hline Condition & \multicolumn{1}{c}{ Menstrual Phase } & Background Noise \\
\hline 1 & Pre-Menstrual (PMS) & Without \\
2 & Pre-Menstrual (PMS) & With $80 \mathrm{~dB}(\mathrm{~A})$ \\
3 & Menstruation (Mens) & Without \\
4 & Menstruation (Mens) & With $80 \mathrm{~dB}(\mathrm{~A})$ \\
5 & Post-Menstruation (PM) & Without \\
6 & Post-Menstruation (PM) & With $80 \mathrm{~dB}(\mathrm{~A})$ \\
\hline
\end{tabular}

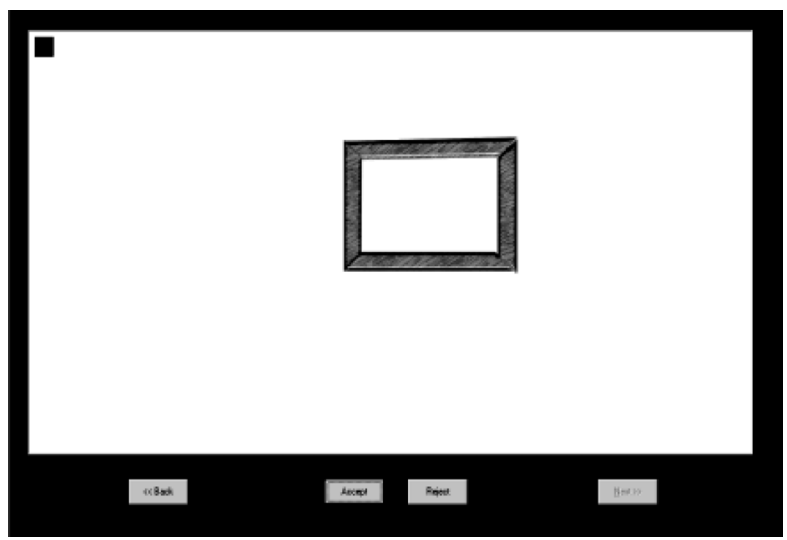

Fig. 1 The area of the inspected object on the VDT screen.

"reject" button (right button) or a non-defective object by pressing an "accept" button (left button). Before performing the visual inspection task, subjects were introduced to the characteristics of each picture that represented defective and non-defective objects. In this session, subjects memorized the visually shown pictures that should be inspected during the visual inspection task on the VDT. After a brief explanation of the task, they practiced the inspection task for five minutes or until they gained less error in the training session.

Visual inspection task performance was measured based on SDT, which is often used in modeling the decision-making process in an inspection task (Jiang et al., 2004). The SDT metrics used in this measurement were sensitivity index $\left(d^{\prime}\right)$ and response criteria index $(\beta)$. Sensitivity refers to the ability of an inspector to discriminate between defective and nondefective objects and response criteria referred to inspector response bias or tendency of an inspector to call an object defective or non-defective. The observed proportion of correct rejection of defective objects (hit) and the proportion of incorrect rejection of non-defective objects (false alarm) were recorded as the basic calculation of SDT metrics $\left(d^{\prime}\right.$ and $\beta$ ). The $d^{\prime}$ was calculated using the equation $d^{\prime}=\mathrm{Z}($ hit $)-\mathrm{Z}(\mathrm{FA})$, where $\mathrm{Z}$ (hit) and $\mathrm{Z}(\mathrm{FA})$ were standard normal deviates corresponding to the proportion of hits and false alarms, while the $\beta$ was measured by the ratio of two ordinates of $Z$ (hit) and $\mathrm{Z}$ (FA).

After the visual inspection session, subjects were asked to fill in a subjective mental workload questionnaire using the NASA TLX questionnaire (Hart and Staveland, 1988), which

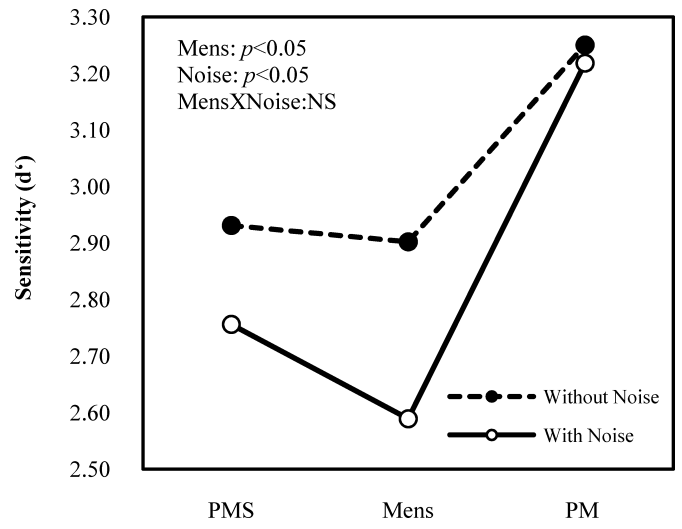

Fig. 2 Interaction plot between menstrual cycle and noise on the $d^{\prime}$.

is commonly used to measure task workload. The subjects had to rate six different workload aspects ("mental demand," "physical demand," "time demand," "performance," "effort," and "frustration level") on scales from "low" to "high." After completion of the NASA TLX questionnaire, subjects were asked to compare the six component of the NASA TLX in all possible pair-wise combination to identify which of the pair contributes more to subjective workload.

\section{Statistical analysis}

The average data of $d^{\prime}, \beta$, and NASA TLX were presented as mean \pm SD. Statistical analysis using Analysis of variance (ANOVA) was utilized to examine the main effect of the menstrual cycle and background noise on task performance and the NASA TLX result and determine which differences were statistically significant differences. The significance level was set at $p<0.05$.

\section{Results}

\section{Sensitivity index $\left(d^{\prime}\right)$}

ANOVA revealed a significant main effect of menstrual phase $(p<0.01)$ and the presence of background noise $(p<0.05)$ on $d^{\prime}$. As shown in the interaction plot between menstrual cycle and noise (Fig. 2), $d^{\prime}$ during PM was higher than during PMS and Mens, both with and without noise, while the Mens condition showed $d^{\prime}$ at the lowest value. Fig. 2 also showed that $d^{\prime}$ was lower when the background noise was presented during visual inspection. Nevertheless, no significant interaction effect between menstrual phase and noise on $d^{\prime}$, which was our main interest in this study, was confirmed.

Regarding the pair-wise comparison between each condition, it indicated that $d^{\prime}$ reached the lowest value at menstruation with the noise condition and reached the highest value at the post-menstrual without noise condition. During the post-menstrual condition, both with and without noise, $d^{\prime}$ was significantly higher than all conditions $(p<0.01$, Table 2$)$ but no significant difference was observed when compared to the PMS without noise condition. Interestingly, during the Mens condition, the $d^{\prime}$ between without noise and with noise 
presented was significantly different in the same condition $(p<0.01)$ while in the PMS and PM condition, there were no significant differences between without and with noise presented.

\section{Response criteria index $(\beta)$}

There were no differences among conditions for either the menstrual phase or background noise, nor were there any interactions between the two factors on $\beta$ (Fig. 3). Thus, an attempt to group the subjects based on $\beta$ was made to look for the possibilities of subject's tendency in decision-making process during visual inspection in all experimental conditions. Based on SDT metrics, the $\beta$ value of all subjects during the PM condition which did not exceed the ideal response criterion $\left(\beta_{\text {opt }}=9.0\right)$ that represents a conservative criterion adopted by the subjects. Conservative criterion means the tendency of a subject to accept a defective object, while the opposite is risky

Table 2 Visual inspection task result based on SDT metrics: sensitivity index $\left(d^{\prime}\right)$ and response criteria index $(\beta)$

\begin{tabular}{cllc}
\hline \multicolumn{2}{c}{ Condition } & \multicolumn{1}{c}{$d^{\prime}$} & $\beta$ \\
\hline 1 & PMS-Without & $2.93 \pm 0.38$ & $15.80 \pm 6.85$ \\
2 & PMS-With & $2.76 \pm 0.37^{*}$ & $14.99 \pm 7.08$ \\
3 & Mens-Without & $2.90 \pm 0.29^{* a}$ & $14.30 \pm 8.78$ \\
4 & Mens-With & $2.59 \pm 0.28^{*}$ & $11.05 \pm 8.17$ \\
5 & PM-Without & $3.25 \pm 0.27$ & $15.17 \pm 5.43$ \\
6 & PM-With & $3.22 \pm 0.24$ & $17.28 \pm 5.54$ \\
\hline
\end{tabular}

$* p<0.01$ compared to PM with noise and PM without noise condition, ${ }^{\mathrm{a}} p<0.01$ between with and without noise during M phase. criteria, obtained when $\beta$ is less than $\beta_{\text {opt }}$, which means the tendency of a subject to reject a non-defective object.

However, as the presence of background noise, two subjects' criteria were inconsistently changed from the conservative criterion to the risky criterion and remained consistently risky during PMS. In addition, during the Mens condition with the presence of noise, six subjects' criteria were the risky criterion, while the other four subjects remained consistent for all conditions (Table 3).

\section{NASA-TLX scores}

The results for the NASA-TLX after the visual inspection task are illustrated in Table 4. For the six scales of NASA-TLX ratings of perceived workload after performing the visual

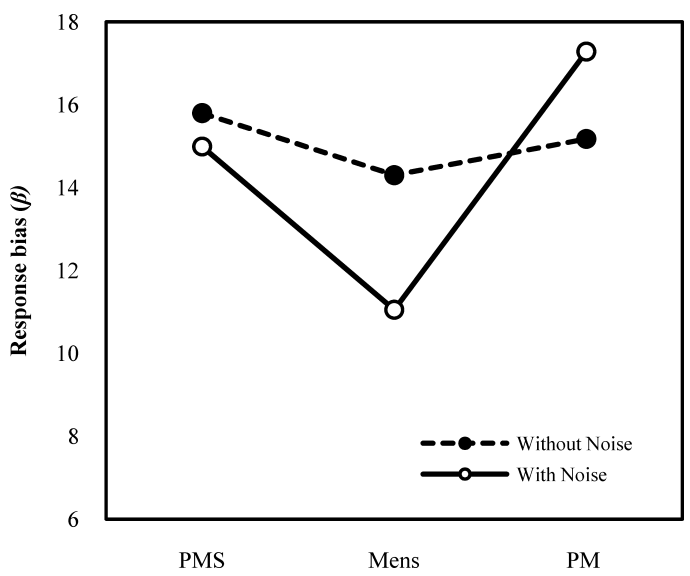

Fig. 3 Interaction plot between menstrual cycle and noise on the $\beta$.

Table 3 Response criteria for each individual subject in all conditions

\begin{tabular}{|c|c|c|c|c|c|c|}
\hline Subject & PMS-Without & PMS-With & Mens-Without & Mens-With & PM-Without & PM-With \\
\hline 1 & Risky & Risky & Risky & Risky & Conservative & Risky \\
\hline 2 & Conservative & Conservative & Conservative & Conservative & Conservative & Conservative \\
\hline 3 & Conservative & Conservative & Conservative & Conservative & Conservative & Conservative \\
\hline 4 & Conservative & Conservative & Conservative & Conservative & Conservative & Conservative \\
\hline 5 & Conservative & Risky & Risky & Risky & Conservative & Conservative \\
\hline 6 & Conservative & Conservative & Conservative & Risky & Conservative & Conservative \\
\hline 7 & Conservative & Conservative & Conservative & Conservative & Conservative & Conservative \\
\hline 8 & Conservative & Conservative & Conservative & Risky & Conservative & Conservative \\
\hline 9 & Conservative & Conservative & Conservative & Risky & Conservative & Conservative \\
\hline 10 & Risky & Risky & Risky & Risky & Conservative & Risky \\
\hline
\end{tabular}

Table 4 NASA Task Load Index (NASA-TLX) of all conditions

\begin{tabular}{|c|c|c|c|c|c|c|c|c|}
\hline & Condition & Mental & $\begin{array}{l}\text { Physical } \\
\text { Demand }\end{array}$ & $\begin{array}{c}\text { Time } \\
\text { Demand }\end{array}$ & $\begin{array}{c}\text { Effort } \\
\text { Demand }\end{array}$ & Performance & Frustration & $\begin{array}{c}\text { AWWL } \\
\text { Level }\end{array}$ \\
\hline 1 & PMS-Without & $68.50 \pm 15.28$ & $31.00 \pm 20.11$ & $63.50 \pm 17.80$ & $58.00 \pm 13.98$ & $67.00 \pm 13.17$ & $55.00 \pm 21.08$ & $62.64 \pm 10.04$ \\
\hline 2 & PMS-With & $71.50 \pm 9.44$ & $37.00 \pm 19.18$ & $60.50 \pm 15.54$ & $63.00 \pm 9.78$ & $67.00 \pm 15.13$ & $55.00 \pm 20.00$ & $65.77 \pm 9.49$ \\
\hline 3 & M-Without & $72.00 \pm 12.95$ & $41.00 \pm 22.58$ & $60.00 \pm 19.44$ & $64.00 \pm 8.43$ & $71.00 \pm 12.65$ & $57.50 \pm 19.18$ & $67.97 \pm 9.17$ \\
\hline 4 & M-With & $75.50 \pm 13.01$ & $44.50 \pm 23.51$ & $68.00 \pm 18.29$ & $71.00 \pm 9.66$ & $68.50 \pm 15.64$ & $68.00 \pm 16.70$ & $73.13 \pm 9.28$ \\
\hline 5 & PM-Without & $62.00 \pm 13.98$ & $30.50 \pm 13.22$ & $55.50 \pm 16.57$ & $52.50 \pm 11.12$ & $64.50 \pm 13.01$ & $50.50 \pm 16.24$ & $56.30 \pm 8.78$ \\
\hline 6 & PM-With & $68.50 \pm 8.83$ & $42.00 \pm 17.35$ & $61.50 \pm 19.16$ & $62.00 \pm 10.85$ & $67.50 \pm 13.79$ & $57.00 \pm 13.17$ & $62.90 \pm 6.70$ \\
\hline
\end{tabular}

\footnotetext{
${ }^{1)}$ Adaptive weighted workload
} 


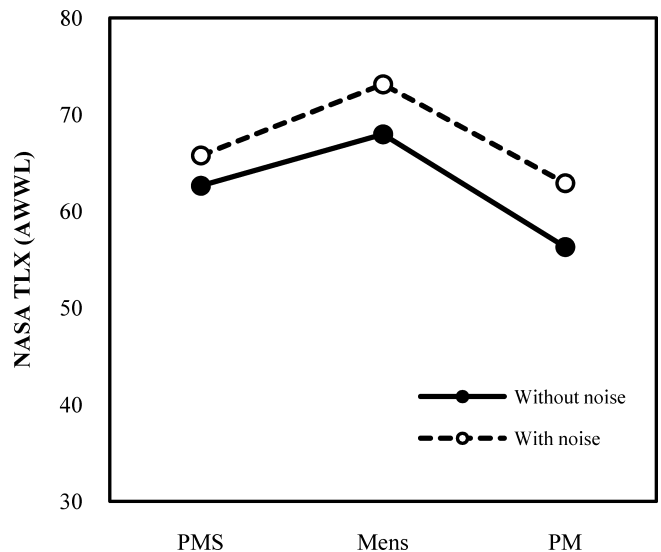

Fig. 4 Interaction plot between menstrual cycle and noise on the NASA TLX (AWWL).

inspection task, there were no significant main effects of menstruation and background noise for "mental demand," "time demand," "effort," "performance," and "frustration level." All ratings scored around 60 points for all conditions and "mental demand" was the highest score among all ratings especially in the Mens with noise condition. Additionally, the overall observation (adaptive weighted workload) showed scores above 60 points except the PM without background noise condition and there were tendencies for AWWL to be higher for the noise presence in all menstruation conditions than for those in the without-noise condition (Fig. 4).

\section{Discussion}

In this study, we hypothesized that visual inspection task performance might be affected by menstrual cycle and background noise, and it was expected that the combinational effect of both factors would exist. However, there was not enough evidence to support our hypothesis that there were combinational effects of the menstrual cycle and the presence of background noise on visual inspection task performance. Our result only indicated that visual inspection task performance was affected by the menstrual cycle $(p<0.01)$ and background noise $(p<0.05)$, without any combinational effect or interaction between menstrual cycle and background noise. Consequently, we analyzed the main effect of menstrual cycle and background noise on visual inspection task performance separately.

The result showed that the ability of subjects in discriminating between defective and non-defective objects was significantly higher during the PM condition, with or without the presence of background noise and significantly lower during the Mens condition with the presence of background noise. This result indicated that the visual inspection task performance was better in the PM condition compared to the PMS and Mens phase; visual inspection task performance was low during the PMS and Mens phase. Diamond et al. (1972) reported that menstrual cycle affected visual detection task performance and suggested that visual functions were affected by cyclic hormonal changes during the menstrual cycle. This result was also consistent with the Ward et al. (1978) findings that visual detection ability fluctuated during the menstrual cycle with impaired performance at the PMS phase. The impaired performance during this period might be caused by the fluctuation of endogenous hormone levels (Ward et al., 1978) and might be associated with sex steroid levels (Philips and Sherwin, 1992). Our results supported the notion that menstrual cycle has an effect on cognitive function but we doubted that this effect was due to hormonal changes during the menstrual cycle. There was a lack of evidence to support the conclusion that changes in hormone levels might affect cognitive abilities, as stated by Gordon and Lee (1993). Nonetheless, we did not include any hormonal level and physiological measurements to prove any relation between hormonal changes and the decrement of visual inspection performance during the PMS and menstruation phase. Thus we cannot say that fluctuation of hormone levels during the menstrual cycle affects cognitive performance in our study.

In addition, this study also showed that the presence of background noise also affected the cognitive functions of subjects as demonstrated by the sensitivity index deterioration when background noise appeared. It was notable that background noise presented during presentation of memorized stimuli might impair working memory. Some other researchers concluded that impairment of noise occurred on some tasks such as detection task (Smith, 1988) and memory (Gomes et al., 1999). We observed that the decrement of detection task performance and working memory was shown by the decrease of the $d^{\prime}$ when background noise presented, especially during the Mens condition. In addition, the presence of noise during the visual inspection task contributed to the higher mental demand shown in the NASA-TLX rating which was attributed to the decrement of the sensitivity index. The result of this study was consistent with Gramopadhye and Wilson (1997), who have shown that the higher value of the sensitivity index in the quiet condition followed by the presence of noises both intermittent and continuous could be attributed to the increased mental workload.

We also considered that the performance decrement on the visual inspection task might be affected by noise exposure time. Frankenhaeuser and Lundberg (1977) reported that time exposure in noise increased the difficulty in concentration. In addition, longer exposure time of noise seemed to produce performance decrement (Gawron, 1982). Therefore, the fact that the visual inspection performance decrement observed in this study was accounted by the noise exposure time, which lasted around 15 to 20 minutes. The length of noise exposure time increased the mental and time demand, which increased the difficulty in concentration and was followed by the decrement of the sensitivity index. In addition, Drury (1992) indicated that the time available for the inspection would affect human performance in the visual search component of the 
inspection; the more time an inspector has to inspect the better the performance in finding defects. Subjects perceived that the presence of background noise seemed to be annoying. Most of them expressed a preference to perform the task as quickly as possible due to noise annoyance. For that reason, we assumed that subjects spent less time inspecting the pictures on the VDT screen that then affected performance negatively. The more the noise exposure, the more annoyed the subject felt. This is in spite of the fact that the average time to make a decision in without noise condition was greater than in the with-noise condition. Thus, we suspected that the presence of noise and time exposure during visual inspection task impaired subject concentration and increased subjective annoyance, which resulted in a performance decrement.

The tendency of subjects in accepting and rejecting inspected objects was not significantly affected by menstrual cycles or by the presence of background noise. This result was consistent with the Nakatani et al. (1993) findings that reported menstrual cycles had no effect on the response criteria index. There were, however, individual differences among subjects that were assumed as one of the strongest factors in this study. It was possible that decision-making performance, shown by the response criteria index, changed over time. Williges (1969) reported that a performance change over time was characterized by the change in the subject's response criterion when the signal-to-noise ratio was low. In our case, the signalto-noise ratio was 1:9 and some subjects shifted their response criteria over time.

Our study showed that several subjects inconsistently changed their tendency from the "conservative" criterion to the "risky" criterion. We grouped these subjects into an inconsistent group. The rest subjects who remained to be consistently in the "conservative" criterion, were grouped into a consistent group. The changes of criteria occurred when background noise was presented. We predicted that subjects within the inconsistent group shifted their criteria from "conservative" to "risky" due to the personality types among subjects. Belojevic et al. (1997) reported that concentration problems were more pronounced in noise among introverted subjects. Another study by Norman and Watson (1976) indicated that extraverts and introverts had different reactions to cognitive consistency; introverts were significantly more likely to change their behaviors in such demanding condition. Thus, we suspected that subjects within the inconsistent group might be predominantly introverts while the subjects from consistent group might be extroverts.

The subjective workload after performing the visual inspection task was not significantly affected by menstrual cycle and the presence of background noise. When we analyzed each item in the NASA-TLX, most ratings scored around 60 and no significant differences were observed among conditions. It showed that the NASA-TLX might be insensitive to the fluctuation in mental workload during the menstrual cycle, as reported by Sato and Miyake (2004). Among all ratings, our findings showed that mental demand was higher than other ratings in all conditions, especially during the menstruation condition with or without the presence of noise. We presumed that visual inspection during menstruation was a mentally demanding task.

From the above, we concluded that the cognitive ability of women during visual inspection might be independently affected by menstrual cycle and the presence of background noise. No combinational effect between menstrual cycle and background noise was confirmed in this study. According to this result we suggest minimizing any visual inspection task during the menstrual period because our results show that performance decreased during the menstruation period. In addition, even though we observed no combinational effect between menstrual cycle and background noise, it is necessary to consider the noise level in the working area as it might affect performance in visual inspection.

No changes in response criteria index in all menstrual cycle conditions and the presence of background noise were observed in this study. The changes of response criteria might depend on the personality types of subjects. The presence of noise might affect the tendency of introverted subjects in detecting objects during a visual inspection task.

However, personality tests and physiological measurements during menstrual cycles were not conducted in our study. On the other hand, our study also found that there was no correlation between subjective workload and sensitivity index as expected before, but introversion of subjects was suspected as an additional factor that might affect visual inspection performance. Thus, we should say that further study, which includes personality tests, physiological indices, and increasing task load, are required to verify and support our findings.

Acknowledgment This study was conducted in the Laboratory of Ergonomics and Work Systems, Department of Mechanical and Industrial Engineering, Gadjah Mada University Indonesia. We would like to thank the female students of Gadjah Mada University who cooperated with this study as volunteer subjects, and their superiors for allowing this study to be performed.

\section{References}

Belojevic G, Jakovljevic B, Aleksic O (1997) Subjective reactions to traffic noise with regard to some personality traits. Environ Int 23: 221-226

Diamond M, Diamond AL, Mast M (1972) Visual sensitivity and sexual arousal levels during the menstrual cycle. J Nerv Ment Dis 155: 170-176

Drury CG (1992) Inspection performance. In Slavendy G ed. Handbook of Industrial Engineering. John Wiley \& Sons, 2282-2314

Frankenhaeuser M, Lundberg V (1977) The influence of cognitive set on performance and arousal under different noise load. Motiv Emot 1: 139-149

Gallwey TJ (1998) Evaluation and control of industrial 
inspection: Part II- the scientific basic for the guide. Int J Ind Ergon 22: 51-65

Gawron VJ (1982) Performance effects of noise intensity, psychological set, and task type and complexity. Hum Factors 24: 225-243

Gomes LMP, Pimenta AJFM, Branco NAC (1999) Effects of occupational exposure to low frequency noise on cognition. Aviat Space Environ Med 70: 115-118

Gordon HW, Lee PA (1993) No difference in cognitive performance between phases of the menstrual cycle. Psychoneuroendocrinology 18: 521-531

Gramopadhye AK, Wilson K (1997) Noise, feedback training, and visual inspection performance. Int $\mathrm{J}$ Ind Ergon 20: 223-230

Hart SG, Staveland LE (1988) Development of NASA-TLX (Task Load Index): Results of experimental and theoretical research. In Hancock PA, Meshkati N eds. Human mental workload. North-Holland, 139-183

Jiang X, Khasawneh MT, Master R, Bowling SR, Gramopadhye AK, Melloy BJ, Grimes L (2004) Measurement of human trust in a hybrid inspection system based on signal detection theory measures. Int $\mathrm{J}$ Ind Ergon 34: 407-419

Kasamatsu K, Suzuki S, Anse M, Funada MF, Idogawa K, Ninomija SP (2002) Menstrual cycle effect on mental arithmetic task performance. J Physiol Anthropol 21: 285290

Nakatani C, Sato N, Matsui M, Matsunami M, Kumashiro M (1993) Menstrual cycle effects on a VDT based simulation task: cognitive indices and subjective rating. Ergonomics 36: 331-339

Norman RMG, Watson LD (1976) Extraversion and reactions to cognitive inconsistency. J Res Pers 10: 446-456

Parsons KC (2000) Environmental ergonomics: a review of principles, method and models. Appl Ergon 31: 581-594

Philips SM, Sherwin BB (1992) Variation in memory function and sex steroid hormones across the menstrual cycle. Psychoneuroendocrinology 17: 497-506

Rosenberg L, Park S (2001) Verbal and spatial functions across the menstrual cycle in healthy young women. Psychoneuroendocrinology 27: 835-841

Sato N, Miyake S (2004) Cardiovascular reactivity to mental stress: relationship with menstrual cycle and gender. J Physiol Anthropol Appl Human Sci 23: 215-223

Smith AP (1988) Acute effects of noise exposure: an experimental investigation of the effect of noise and task parameter on cognitive vigilance task. Int Arch Occup Environ Health 60: 307-310

Smith AP (1991) Noise and aspects of attention. Br J Psychol 82: 313-324

Ward MM, Stone CS, Sandman CA (1978) Visual Perception in women during the menstrual cycle. Physiol Behav 20: 239-243

Warner HD, Heimstra NW (1972) Effect of noise intensity on visual target detection performance. Hum Factors 14: 181185

Williges RC (1969) Within-session criterion changes compared to an ideal observer criterion in a visual monitoring task. J Exp Psychol 81: 61-66

Received: March 10, 2009

Accepted: August 26, 2009

Correspondence to: Titis Wijayanto, Department of Ergonomics, Graduate School of Design, Kyushu University, 4-9-1 Shiobaru, Minami-ku, Fukuoka 815-8540, Japan

Phone: +81-92-553-9493 (Office)

Fax: +81-92-553-4522

e-mail: twijayanto@gmail.com 